\title{
Semi-Analytical Approaches to Local Electroweak Baryogenesis
}

\author{
Arthur Lue ${ }^{1 *}$, Krishna Rajagopal ${ }^{2 \dagger}$ and Mark Trodden ${ }^{1 \ddagger}$ \\ ${ }^{1}$ Center for Theoretical Physics \\ Laboratory for Nuclear Science and Department of Physics \\ Massachusetts Institute of Technology, Cambridge, Massachusetts 02139, USA. \\ ${ }^{2}$ Lauritsen Laboratory of High Energy Physics \\ California Institute of Technology, Pasadena, CA 91125, USA.
}

\begin{abstract}
We examine two semi-analytical methods for estimating the baryon asymmetry of the universe (BAU) generated in scenarios of "local" electroweak baryogenesis (in which the requisite baryon number violation and $\mathcal{C P}$ violation occur together in space and time). We work with the standard electroweak theory augmented by the addition of a $\mathcal{C P}$ violating dimension six operator. We work in the context of a first order phase transition, but the processes we describe can also occur during the evolution of a network of topological defects. Both the approaches we explore deal with circumstances where the bubble walls which convert the high temperature phase to the low temperature phase are thin and rapidly moving. We first consider the dynamics of localized configurations with winding number one which remain in the broken phase immediately after the bubble wall has passed. Their subsequent decay can anomalously produce fermions. In a prelude to our analysis of this effect, we demonstrate how to define the $\mathcal{C}$ and $\mathcal{C P}$ symmetries in the bosonic sector of the electroweak theory when configurations with nonzero winding are taken into account. Second, we consider the effect of the passage of the wall itself on configurations which happen to be near the crest of the ridge between vacua as the wall arrives. We find that neither of the simple approaches followed here can be pushed far enough to obtain a convincing estimate of the BAU which is produced. A large scale numerical treatment seems necessary.
\end{abstract}

MIT-CTP-2590

CALT-68-2089

\footnotetext{
*ithron@mit.edu

†krishna@theory.caltech.edu

†trodden@ctpa04.mit.edu. Also, Visiting Scientist, Brown University, Providence, RI 02912.
} 


\section{INTRODUCTION}

One of the central challenges of modern cosmology is to explain how the baryon asymmetry of the universe (BAU) can be dynamically generated from baryon number symmetric initial conditions. The relevant observational quantity is the baryon to entropy ratio which is tightly constrained by primordial nucleosynthesis to lie in the interval $2 \times 10^{-10}<n_{B} / s<5 \times 10^{-10}$. Sakharov [1] showed that there are three necessary conditions which must be satisfied if a particle physics model is to produce a net cosmological baryon asymmetry. These are a violation of baryon number $(\mathcal{B})$ conservation, a departure from thermal equilibrium, and violations of charge conjugation $(\mathcal{C})$ and charge-parity $(\mathcal{C P})$ symmetries. The standard model of electroweak interactions includes all three ingredients, as we discuss below. This observation has led to the suggestion that the standard model might be responsible for the generation of the baryon asymmetry of the universe [2 16].

Baryon number is exactly conserved at the classical level in the standard electroweak theory, but at the quantum level this ceases to be true as a consequence of the anomaly [17]. The relevant process at zero temperature is an exponentially suppressed tunnelling under the energy barrier which separates inequivalent vacua in gauge and Higgs field configuration space. The lowest point on the crest of the barrier is a saddle point configuration called the sphaleron [18]. At nonzero temperature and in particular for temperatures around or above the critical temperature of the electroweak phase transition it is possible for thermal effects to cause classical transitions over the sphaleron barrier [3, 19]. Although at temperatures above the electroweak phase transition anomalous baryon number and lepton number $(\mathcal{L})$ violation is unsuppressed in the standard model, this occurs in such a way that $\mathcal{B}-\mathcal{L}$ is conserved. Thus, any preexisting $\mathcal{B}-\mathcal{L}$ asymmetry cannot be erased by electroweak processes. We are assuming that above the temperature of the electroweak phase transition $\mathcal{B}$ and $\mathcal{L}$ are both zero.

In order to construct a scenario for electroweak baryogenesis we must achieve a departure from thermal equilibrium. In the context of the early universe this is typically satisfied in one 
of two ways. One possibility is that the electroweak phase transition is considered to be first order so that the violent conversion of the high temperature phase to the low temperature phase results in non-equilibrium conditions near the bubble walls separating the phases [5 [14]. An alternative is that non-equilibrium processes occur in topological defects, such as cosmic strings, around which the electroweak symmetry is restored [15]. In defect scenarios, the phase boundaries between the interior and exterior of the defects behave in an analogous manner to bubble walls. In this paper, we shall phrase our considerations entirely in terms of bubble walls at a first order phase transition, even though the processes we discuss also arise in defect mediated baryogenesis.

We make several standard assumptions related to the rate per unit volume $\Gamma$ for baryon number violating processes in thermal equilibrium, which we write as

$$
\Gamma=\kappa\left(\alpha_{W} T\right)^{4}
$$

We assume that the phase transition is strongly enough first order that at temperatures below $T_{c}, \Gamma$ is small enough that the BAU created at the transition is not erased by subsequent electroweak baryon number violating processes. (The constraint turns out to be $\kappa<10^{-9}$ for $T<T_{c}[20,21]$.) It now seems likely that this requires extending the field content of the theory in some way which makes the phase transition more strongly first order. As is often done, we use the minimal standard model with a Higgs mass lighter than is allowed by experiment to model the strongly first order phase transition in the extended theory. We also assume, again as is standard, that at temperatures just above $T_{c}$ the dimensionless quantity $\kappa$ is not very small.

Sakharov's third condition is that $\mathcal{C}$ and $\mathcal{C P}$ must be violated. The standard electroweak theory is maximally $\mathcal{C}$ violating due to the $\mathrm{V}$-A nature of the interactions. As we will show, the purely bosonic sector of the theory is also $\mathcal{C}$ violating once topological properties of the sector are treated correctly. The only violation of $\mathcal{C P}$ in the electroweak sector of the standard model occurs in the CKM matrix. Electroweak baryogenesis scenarios using this source of $\mathcal{C P}$ violation are generally expected to produce a BAU which is far too small. We 
can also ignore the $\mathcal{C P}$ violating $\theta$ term of QCD since this is known to be tiny. Therefore, if we are to generate an appreciable baryon asymmetry it is necessary to consider extending the standard model to include new sources of $\mathcal{C P}$ violation. A popular approach has been to consider a two-Higgs doublet model with explicit renormalizable $\mathcal{C P}$ violating terms in the Higgs sector [6, 8, 9, 12, 13, 15]. This enhances the $\mathcal{C P}$ violation beyond that present in the standard model but adds a host of new free parameters to the theory. Here we follow the simpler approach first discussed by Dine et al. [7]. We assume that there is some $\mathcal{C P}$ violating physics (beyond that in the standard model) at an energy scale $M$ greater than $v=250 \mathrm{GeV}$. At scales less than $M$, the effective theory is the standard model plus nonrenormalizable operators, some of which are $\mathcal{C P}$ odd. In this paper, for definiteness, we add the dimension six $\mathcal{C P}$ odd operator

$$
\mathcal{O}=\frac{b}{M^{2}} \operatorname{Tr}\left(\Phi^{\dagger} \Phi\right) \operatorname{Tr}\left(F_{\mu \nu} \tilde{F}^{\mu \nu}\right)
$$

to the standard model Lagrangian density, and do not augment the field content of the theory. Here $\Phi$ is the Higgs field defined in (2.3), $F_{\mu \nu}$ is the $S U(2)$ field strength tensor defined in (2.2) and

$$
\tilde{F}^{\mu \nu}=\frac{1}{2} \epsilon^{\mu \nu \alpha \beta} F_{\alpha \beta}
$$

is the dual of the field strength tensor. $\mathcal{O}$ is the lowest dimension $\mathcal{C P}$ odd operator which can be constructed from minimal standard model Higgs and gauge fields. Standard model $\mathcal{C P}$ violation in the CKM matrix does induce the term $\mathcal{O}$ with $M=v$ in the effective action, but the coefficient $b$ is thought to be tiny. For us, $b$ is a dimensionless coupling measuring the strength of the $\mathcal{C P}$ violating physics at the scale $M$, above which our effective theory ceases to be valid. Throughout most of this paper, $b$ and $M$ occur only in the combination $b / M^{2}$, and in this sense we are introducing a single new parameter.

The operator $\mathcal{O}$ induces electric dipole moments for the electron and the neutron, and the strongest experimental constraint on the size of such an operator come from the fact that such dipole moments have not been observed. Working to lowest order (one-loop) we find 


$$
\frac{d_{e}}{e}=\frac{m_{e} \sin ^{2}\left(\theta_{W}\right)}{8 \pi^{2}} \frac{b}{M^{2}} \ln \left(\frac{M^{2}+m_{H}^{2}}{m_{H}^{2}}\right) .
$$

$M^{2}$ arises in the logarithm without $b$ because $M$, the scale above which the effective theory is not valid, is the ultraviolet cutoff for the divergent loop integral. A result similar to (1.4) was obtained in Ref. [22]. Using the experimental limit [23] $d_{e} / e<4 \cdot 10^{-27} \mathrm{~cm}$ we find the bound

$$
\frac{b}{M^{2}} \ln \left(\frac{M^{2}+m_{H}^{2}}{m_{H}^{2}}\right)<\frac{1}{(3 \mathrm{TeV})^{2}} .
$$

The experimental limit 24] on the neutron electric dipole moment $d_{n}$ is weaker than that on $d_{e}$, but because $d_{n}$ is proportional to the quark mass rather than to the electron mass, the constraint obtained using $d_{n}$ is comparable to (1.5). A baryogenesis scenario which relies on $\mathcal{C P}$ violation introduced via the operator $\mathcal{O}$ must respect the bound $(1.5)$.

We wish to estimate the BAU generated during a first order electroweak phase transition in the minimal standard model with the addition of the operator $\mathcal{O}$ with a coefficient $b / M^{2}$ satisfying (1.5). Baryogenesis can occur either "locally" (baryons are produced as a result of $\mathcal{B}$ violating processes and $\mathcal{C P}$ violating processes occuring together near bubble walls) or "nonlocally" (baryons are produced as a result of $\mathcal{C P}$ violation occurring as particles bounce off bubble walls and $\mathcal{B}$ violation occuring away from bubble walls in the high temperature phase). In general, both local and nonlocal baryogenesis will occur and the BAU will be the sum of that generated by the two processes. We have nothing to add to the existing treatments [5,9 14 of nonlocal baryogenesis, also called baryogenesis by charge transport. In this paper, we reconsider the models of baryogenesis first introduced by Turok and Zadrozny [6] and Dine et al. [7], and try to estimate the BAU produced locally. Throughout this paper, we assume that the time during which the expectation value of the Higgs field is changing at any point in space is short compared to all other timescales in the problem. Throughout, we refer to walls satisfying this criterion as "thin", even though they may meet the criterion either by being thin enough or by moving fast enough. It is worth noting that for walls which move at a supersonic velocity, diffusion from the wall foward into the high temperature phase, 
and hence nonlocal baryogenesis, are not possible. In the opposite limit to that we treat, that is if the walls are "thick" in the sense that the Higgs field changes slowly relative to all other timescales, then the effects of the operator $\mathcal{O}$ can be treated approximately as a chemical potential for baryon number [7], realizing a possibility first considered in Ref. [4] and called spontaneous baryogenesis. The local contribution to the BAU in the thick wall limit was first estimated by Dine et al. [0] and has been further analyzed in Refs. [8, 11, 12], and is not affected by the considerations of this paper.

In this paper we examine two semi-analytical methods for estimating the efficiency of local baryogenesis occurring in the standard model with the addition of the operator $\mathcal{O}$, assuming a strongly first order phase transition and thin bubble walls. In section III, we explore an approach pioneered by Turok and Zadrozny [6] to estimate the baryon asymmetry by considering the relaxation of topologically nontrivial field configurations produced during the phase transition. In contrast with Ref. [6] we study the full Higgs plus gauge dynamics and, because we introduce $\mathcal{C P}$ violation via the operator $\mathcal{O}$ rather than using the two Higgs doublet model employed in Ref. [6], we need not and do not add new fields to the theory. Turok and Zadrozny considered effects which occur while a (thick) bubble wall is passing. We use their method to consider physics after the passage of a thin wall. Unfortunately, we show that the use of a one-parameter family of spherically symmetric configurations as in Ref. [6] can give qualitatively misleading results, and demonstrate the difficulty of obtaining analytic estimates in this picture without doing a full scale numerical simulation. In Section II we discuss certain properties of gauge and Higgs field configurations in the standard electroweak theory, as a prelude to our discussion of baryon number production in Section III. Included in Section II is a demonstration that when the $\mathcal{C}$ and $\mathcal{P}$ transformations are defined to treat configurations with winding properly, the bosonic sector of the electroweak lagrangian is not $\mathcal{C}$ or $\mathcal{P}$ invariant but is $\mathcal{C P}$ invariant. In most treatments, $\mathcal{C}$ violation arises because of the $V-A$ nature of the fermionic part of the theory, so it is amusing to see that even in the bosonic sector, $\mathcal{C}$ is not a good symmetry. In Section IV we turn to a method introduced by Dine et al. [7]. We consider configurations which happen to be 
near the crest of the ridge between vacua as the wall arrives, and estimate the extent to which their velocity in configuration space is modified by the operator $\mathcal{O}$ during the passage of the wall. If the wall is thick, it turns out that the velocity of motion in configuration space is not affected. The asymmetry arises because $\mathcal{O}$ affects the potential energy surface in configuration space during the passage of the wall. If the wall is thin, as we assume in this paper, there is no significant time during which the potential energy surface is affected, but the configuration space velocities are affected asymmetrically. We show how to estimate the quantities entering the final result in the thin wall limit correctly. Nevertheless, we argue that difficulties of the kind encountered in Section III also apply to the method of Section IV, rendering the estimate for the BAU more of an upper bound than an estimate. Thus, it seems to us that neither approach can be pushed far enough to obtain a convincing semi-analytical estimate of the BAU produced by local electroweak baryogenesis in the thin wall limit, and a large scale numerical simulation is called for.

\section{RELEVANT PROPERTIES OF THE STANDARD ELECTROWEAK THEORY}

In this section we examine certain properties of the standard model which are important for the arguments of Section III. Although our motivation is to set the stage for the next section, we have also endeavoured to write this section in such a way that it is independent of the rest of this paper. The relevant dynamics take place in the bosonic sector. We consider fermion production in the background of the evolving Higgs and gauge fields. We do not take into account the back-reaction of the fermions on the bosonic fields. Thus, we begin by looking at the purely bosonic part of the standard model where, for simplicity, we ignore the $U(1)$ hypercharge gauge field.

$$
\mathcal{L}=-\frac{1}{2} \operatorname{Tr}\left(F_{\mu \nu} F^{\mu \nu}\right)-\frac{1}{2} \operatorname{Tr}\left(D^{\mu} \Phi\right)^{\dagger} D_{\mu} \Phi-\frac{\lambda}{4}\left[\operatorname{Tr}\left(\Phi^{\dagger} \Phi\right)-v^{2}\right]^{2},
$$

where

$$
\begin{aligned}
F_{\mu \nu} & =\partial_{\mu} A_{\nu}-\partial_{\nu} A_{\mu}-i g\left[A_{\mu}, A_{\nu}\right] \\
D_{\mu} \Phi & =\left(\partial_{\mu}-i g A_{\mu}\right) \Phi
\end{aligned}
$$


with $A_{\mu}=A_{\mu}^{a} \tau^{a} / 2$ where $\tau^{a}$ are the three Pauli matrices. The standard Higgs doublet $\varphi=\left(\varphi_{1}, \varphi_{2}\right)$ is related to the matrix $\Phi$ by

$$
\Phi(\mathbf{x}, t)=\left(\begin{array}{cc}
\varphi_{2}^{*} & \varphi_{1} \\
-\varphi_{1}^{*} & \varphi_{2}
\end{array}\right) .
$$

Here $v=247 \mathrm{GeV}$ and $g=0.65$. The gauge boson mass is $m_{W}=\frac{1}{2} g v$ and the Higgs boson mass is $m_{H}=\sqrt{2 \lambda} v$.

Note that

$$
\Phi^{\dagger} \Phi=\left(\varphi_{1}^{*} \varphi_{1}+\varphi_{2}^{*} \varphi_{2}\right)\left(\begin{array}{ll}
1 & 0 \\
0 & 1
\end{array}\right)
$$

so that we can write

$$
\Phi=\frac{\sigma}{\sqrt{2}} U
$$

where $\sigma^{2}=2\left(\varphi_{1}^{*} \varphi_{1}+\varphi_{2}^{*} \varphi_{2}\right)=\operatorname{Tr} \Phi^{\dagger} \Phi$, and $U$ is an $S U(2)$ valued field which is uniquely defined at any spacetime point where $\sigma$ does not vanish. Without loss of generality we impose the condition that at all times

$$
\begin{gathered}
\lim _{|\mathbf{x}| \rightarrow \infty} \sigma(\mathbf{x}, t)=v, \\
\lim _{|\mathbf{x}| \rightarrow \infty} U(\mathbf{x}, t)=\left(\begin{array}{ll}
1 & 0 \\
0 & 1
\end{array}\right) .
\end{gathered}
$$

In $A_{0}=0$ gauge, a vacuum configuration is of the form

$$
\begin{aligned}
\Phi & =\frac{v}{\sqrt{2}} U \\
A_{j} & =\frac{1}{i g} \partial_{j} U U^{\dagger} .
\end{aligned}
$$

At any time $t$ when $\sigma(\mathbf{x}, t) \neq 0$ for all $\mathbf{x}$ we have that $U(\mathbf{x}, t)$ is a map from $\mathbf{R}^{3}$ with the points at infinity identified, that is $S^{3}$, into $S U(2)$ and therefore $U(\mathbf{x}, t)$ can be associated with an integer-valued winding

$$
N_{H}(t)=w[U]=\frac{1}{24 \pi^{2}} \int d^{3} x \epsilon^{i j k} \operatorname{Tr}\left[U^{\dagger} \partial_{i} U U^{\dagger} \partial_{j} U U^{\dagger} \partial_{k} U\right],
$$


the Higgs winding number. If $\Phi(\mathbf{x}, t)$ evolves continuously in $t$ then $N_{H}(t)$ can change only at times when there is a zero of $\sigma$ at some point in space. At such times, $N_{H}$ is not defined; at all other times, it is integer-valued. Note that the Higgs winding number of a vacuum configuration (2.8) is equal to its Chern-Simons number

$$
N_{C S}(t)=\frac{g^{2}}{32 \pi^{2}} \int d^{3} x \epsilon^{i j k} \operatorname{Tr}\left(A_{i} \partial_{j} A_{k}+\frac{2}{3} i g A_{i} A_{j} A_{k}\right) .
$$

For a general non-vacuum configuration the Chern-Simons number is not integer-valued.

\section{A. Topologically Interesting Configurations}

In this section we are interested in the dynamics of nonzero energy configurations with nonzero Higgs winding. A simple example is

$$
\begin{aligned}
\Phi(\mathbf{x}) & =\frac{v}{\sqrt{2}} U_{[1]}(\mathbf{x}) \\
A_{\mu}(\mathbf{x}) & =0
\end{aligned}
$$

where $U_{[1]}(\mathbf{x})$ is a winding number one map, say,

$$
U_{[1]}(\mathbf{x})=\exp (i \eta(r) \boldsymbol{\tau} \cdot \hat{\mathbf{x}})
$$

with $\eta(0)=-\pi$ and $\eta(\infty)=0$. The configuration (2.11) has no potential energy but does carry gradient energy because the covariant derivatives $D_{i} \Phi$ do not vanish. This configuration has $N_{H}=1$. If the configuration (2.11) were released from rest it would radiate away its energy and relax towards a vacuum configuration. There are two very different ways for

this to occur [6]. If the characteristic size of $U_{[1]}$ is large compared to $m_{W}^{-1}$, then the gauge field will evolve until it lines up with the Higgs field making the covariant derivatives zero, and at late times $N_{H}$ will still be one. If the characteristic size is small the configuration will shrink, the Higgs field $\sigma$ will go through a zero, and at late times $N_{H}$ will be zero. This dynamics is the subject of the next section.

Note that $N_{H}$ is not invariant under large gauge transformations. However, the change in Higgs winding, $\Delta N_{H}$, is gauge invariant and the two distinct relaxation processes are 
distinguished by whether $\Delta N_{H}$ is zero or nonzero. Throughout this section we choose the gauge such that our prototypical initial configuration is of the form (2.11) which has $N_{H}=1$.

The configuration (2.11) is similar to the Skyrmion which is a winding number one soliton in the nonlinear sigma model associated with QCD. The Skyrme lagrangian has a four derivative interaction which is not present in (2.1). This term stabilizes the soliton because it prevents the winding number one configurations from shrinking to zero size. Note that in the Skyrme model winding number one solitons are identified with baryons and winding number minus one solitons are viewed as antibaryons.

We could view the action (2.1) as an effective theory which describes the low energy degrees of freedom of some more fundamental theory such as technicolor. The sigma model of (2.1) would be to technicolor what the usual sigma model which describes pions is to QCD. The addition of a sufficiently large Skyrme-like term would result in classically stable electroweak solitons which we could call techni-skyrmions. The Higgs winding of such an electroweak soliton would be identified with its technibaryon number. In this paper we are not modifying (2.1) except for the addition of the dimension six $\mathcal{C P}$ violating term (1.2) and all configurations with winding are unstable.

\section{B. Fermion Production}

A wound up configuration of the form (2.11) is not stable and if released from rest it will evolve to a vacuum configuration of the form (2.8) plus radiation. In the process fermions may be anomalously produced. If the fields relax to the vacuum by changing the Higgs winding then there is no anomalous fermion number production. However, if there is no net change in Higgs winding during the evolution (for example $\sigma$ never vanishes) then there is anomalous fermion number production.

To understand these claims consider two sequences of configurations beginning with the wound up configuration (2.11) and ending at the classical vacuum (2.8). The first sequence

ends at the vacuum (2.8) with $U=1$ while the second ends up at $U=U_{[1]}$. Note that these sequences cannot be solutions to the classical equations of motion since the initial 
configurations carry energy whereas the final ones do not. Throughout both sequences we maintain the boundary conditions (2.6) and (2.7). For the first sequence, $\sigma$ must vanish at some intermediate configuration since the Higgs winding changes. For the second sequence, the change in Higgs winding is zero and $\sigma$ need not vanish.

Now introduce an $S U(2)_{L}$ weak fermionic doublet, $\psi$. The fermion is given mass through the usual gauge invariant coupling to the Higgs field $\Phi$ and for simplicity we assume that both the up and down components of the doublet have the same mass, $m$. The fermion field is quantized in the background of the bosonic fields given by our interpolation.

Now, the anomaly equation

$$
\partial_{\mu} J^{\mu}=\frac{g^{2}}{32 \pi^{2}} \operatorname{Tr}(F \tilde{F})
$$

when integrated, implies that the change in the fermion number from the beginning to the end of a sequence is given by

$$
\left.\int d^{3} x J^{0}\right|_{\text {final }}-\left.\int d^{3} x J^{0}\right|_{\text {initial }}=-w[U]
$$

where $U$ is that of the final configuration (2.8). For the first sequence $w$ is one whereas for the second it is zero. Thus fermion number is violated in processes for which the configuration (2.11) unwinds via gauge unwinding, but is not violated when such a configuration unwinds via a Higgs unwinding.

For both of the interpolations which we have considered, the final background configuration is a vacuum configuration of the form (2.8). In this background the lowest energy fermion state has fermion number zero and the fermion number of any other state with $n \psi$ particles and $m \psi$ antiparticles is $n-m$. However, the fermion number of the initial state, where the background has the non-vacuum form (2.11), is more complicated. Suppose that the winding number one map $U_{[1]}$ in 2.11) has a characteristic size $L$. It is known that if $m L \gg 1$ then the lowest energy fermion state in the presence of this background has fermion number one [25 27]. In this sense the configuration (2.11) is said to carry fermion number. If our initial fermion state is this lowest energy state and we let this state evolve 
in the changing background of our first interpolation then the final state will contain one net $\psi$ particle. Although a $\psi$ particle must be produced, there is no violation of fermion number since both initial and final states have fermion number one. If we evolve the same initial state in the background of the second interpolation there will be no net $\psi$ particles in the final state. This is consistent with anomalous fermion production as described by equation (2.14). On the other hand, if we begin with the configuration (2.11) and $m L \ll 1$, then the lowest energy state in this background has fermion number zero. If this state evolves in the background of the first interpolation then there are no net $\psi$ particles in the final state whereas if it evolves in the background of the second interpolation there is one net antifermion produced. This discussion of where the fermion number resides does not alter the general conclusion which is that if we smoothly interpolate from (2.11) to (2.8) with $U=\mathbf{1}$ then there is no anomalous fermion production in this background whereas if we end up at (2.8) with $U=U_{[1]}$ then there is anomalous fermion production.

Of course, we are actually interested in the dynamical evolution of configurations such as (2.11) which are released from rest and end up as outgoing radiation. In this case it is dangerous to use the anomaly equation (2.13) since $\int d^{4} x \operatorname{Tr}(F \tilde{F})$ is not well-defined as an integral and any answer can be obtained for the change in fermion number [28]. Nonetheless, the results of our previous discussion still apply. If the configuration (2.11) is released and falls apart without ever going through a zero of the Higgs field, then the analysis of Ref. 29 is directly applicable and we conclude that one net antifermion is produced just as we did with the second of our interpolations. If the Higgs field unwinds by going through a zero, then one can use arguments presented in Refs. [28,29] to demonstrate that the presence of outgoing radiation in the final configuration does not affect the result above, namely that there is no fermion number violation.

\section{Discrete Symmetries: $\mathcal{C}, \mathcal{P}$ and $\mathcal{C P}$}

In this subsection we study the properties of the lagrangian (2.1) under the discrete transformations $\mathcal{C}, \mathcal{P}$ and $\mathcal{C P}$. We must keep in mind that certain configurations of $\Phi$ 
can be associated with a particle number which equals the Higgs winding defined by (2.9) and (2.5). With the identification of winding with particle number, we will see that (2.1) is not invariant under $\mathcal{C}$ and $\mathcal{P}$ separately but is invariant under $\mathcal{C P}$.

First let us describe the parity transformation. Let $\mathcal{P}^{\prime}$ be the obvious discrete transformation defined as

$$
\begin{aligned}
& \mathcal{P}^{\prime}: A_{0}(\mathbf{x}, t) \rightarrow A_{0}(-\mathbf{x}, t) \\
& \mathcal{P}^{\prime}: A_{i}(\mathbf{x}, t) \rightarrow-A_{i}(-\mathbf{x}, t) \\
& \mathcal{P}^{\prime}: \Phi(\mathbf{x}, t) \rightarrow \Phi(-\mathbf{x}, t)
\end{aligned}
$$

Under the parity transformation $\mathcal{P}^{\prime}$ the lagrangian (2.1) is invariant. However, under $\mathcal{P}^{\prime}$ the winding number given by (2.9) changes sign and we certainly do not want particle number to change sign under parity! Now parametrize the unitary matrix $U$ appearing in (2.5) by the fields $\pi^{a}(\mathbf{x}, t)$ which are weak scale sigma model analogs of the low energy pions

$$
\Phi(\mathbf{x}, t)=\frac{\sigma(\mathbf{x}, t)}{\sqrt{2}} \exp \left(i \tau^{a} \pi^{a}(\mathbf{x}, t)\right)
$$

We can define a parity operator $\mathcal{P}$ which takes $\pi^{a}(\mathbf{x}, t)$ to $-\pi^{a}(-\mathbf{x}, t)$ which is the conventional transformation property of the ordinary pions. Consistent with this we define

$$
\begin{aligned}
& \mathcal{P}: A_{0}(\mathbf{x}, t) \rightarrow A_{0}(-\mathbf{x}, t) \\
& \mathcal{P}: A_{i}(\mathbf{x}, t) \rightarrow-A_{i}(-\mathbf{x}, t) \\
& \mathcal{P}: \Phi(\mathbf{x}, t) \rightarrow \Phi^{\dagger}(-\mathbf{x}, t)
\end{aligned}
$$

With this definition of parity, which we adopt, the winding $w[U]$ is unchanged by a parity transformation. However, in this case the weak interaction lagrangian (2.1) is not parity invariant.

Now turn to charge conjugation. If we include the $U(1)$ interaction we see that $A_{\mu}^{3}$ is a linear combination of the photon and the $Z$-boson. Since the photon is charge conjugation odd we certainly want $A_{\mu}^{3} \rightarrow-A_{\mu}^{3}$. Similarly we want $W_{\mu}^{+} \rightarrow-W_{\mu}^{-}$and $W_{\mu}^{-} \rightarrow-W_{\mu}^{+}$ which is the same as $A_{\mu}^{1} \rightarrow-A_{\mu}^{1}$ and $A_{\mu}^{2} \rightarrow A_{\mu}^{2}$. This is equivalent to the requirement that 
the matrix $A_{\mu}=A_{\mu}^{a} \tau^{a} / 2$ transforms into $\tau_{2} A_{\mu} \tau_{2}$. Thus we can attempt to define charge conjugation by $\mathcal{C}^{\prime}$ where

$$
\begin{aligned}
& \mathcal{C}^{\prime}: A_{\mu} \rightarrow \tau_{2} A_{\mu} \tau_{2} \\
& \mathcal{C}^{\prime}: \Phi \rightarrow \tau_{2} \Phi \tau_{2}
\end{aligned}
$$

This transformation has the property that $\varphi_{1} \rightarrow \varphi_{1}^{*}$ and $\varphi_{2} \rightarrow \varphi_{2}^{*}$ which are the expected transformations of the complex fields and furthermore leaves the lagrangian (2.1) invariant. Note that the Lagrangian (2.1) is invariant under the transformation $\Phi \rightarrow B^{\dagger} \Phi B, A_{\mu} \rightarrow$ $B^{\dagger} A_{\mu} B$, where $B$ is a spacetime independent $S U(2)$ matrix. With $B=i \tau_{2}$ we obtain the transformation (2.18). Thus, the transformation $\mathcal{C}^{\prime}$ is one element of a continuous global symmetry group and should not be viewed as a discrete transformation. In addition, under $\mathcal{C}^{\prime}$ the winding number is unchanged which is unacceptable.

Return to the parametrization of $\Phi$ given by (2.16). Under charge conjugation we expect $\left(\pi^{1}, \pi^{2}, \pi^{3}\right) \rightarrow\left(\pi^{1},-\pi^{2}, \pi^{3}\right)$. To be consistent with this let us define

$$
\begin{aligned}
& \mathcal{C}: A_{\mu} \rightarrow \tau_{2} A_{\mu} \tau_{2} \\
& \mathcal{C}: \Phi \rightarrow \tau_{2} \Phi^{\dagger} \tau_{2}
\end{aligned}
$$

Adopting this definition we have that the winding flips sign under charge conjugation. However, the weak interaction lagrangian (2.1) is not $\mathcal{C}$ invariant.

Consider $\mathcal{C P}$ defined as the composition of (2.17) and (2.19):

$$
\begin{aligned}
& \mathcal{C P}: A_{0}(\mathbf{x}, t) \rightarrow \tau_{2} A_{0}(-\mathbf{x}, t) \tau_{2} \\
& \mathcal{C P}: A_{i}(\mathbf{x}, t) \rightarrow-\tau_{2} A_{i}(-\mathbf{x}, t) \tau_{2} \\
& \mathcal{C P}: \Phi(\mathbf{x}, t) \rightarrow \tau_{2} \Phi(-\mathbf{x}, t) \tau_{2}
\end{aligned}
$$

All fields transform as expected, for example $\varphi_{1}(\mathbf{x}, t) \rightarrow \varphi_{1}^{*}(-\mathbf{x}, t)$, the lagrangian (2.1) is invariant under $\mathcal{C P}$ and the Higgs winding changes sign. Note that $\mathcal{C P}=\mathcal{C}^{\prime} \mathcal{P}^{\prime}$. In defining $\mathcal{C}$ and $\mathcal{P}$ one must be careful to ensure that winding flips under $\mathcal{C}$ but not under $\mathcal{P}$. In so doing, one discovers that with appropriate definitions of $\mathcal{C}$ and $\mathcal{P}$ the bosonic sector of the electroweak lagrangian is not $\mathcal{C}$ or $\mathcal{P}$ invariant but is $\mathcal{C P}$ invariant. 


\section{LOCAL BARYOGENESIS THROUGH UNWINDING}

In this section we explore the possibility of using the method of Turok and Zadrozny [6] to estimate the baryon asymmetry produced by local baryogenesis in a scenario in which the electroweak phase transition is strongly first order and the bubble walls are thin. The

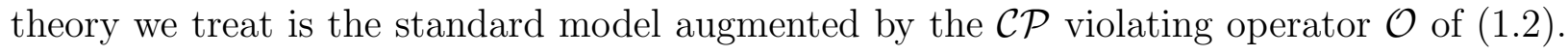
Turok and Zadrozny [6] studied the classical dynamics of topologically nontrivial gauge and Higgs field configurations in the presence of $\mathcal{C P}$ violation. Following Ref. [6], we begin by considering spherically symmetric nonvacuum configurations of the form (2.11) with Higgs winding $N_{H}= \pm 1$ and discuss their dynamics when they are released from rest and evolve according to the equations of motion. Solutions to the equations of motion typically approach a vacuum configuration uniformly throughout space at late times, and these solutions are no exception. There are, however, two qualitatively different possible outcomes of this evolution. One possibility is that at some time during the evolution, $\sigma$, the magnitude of the Higgs field, is zero at some point in space and at late times the configuration tends toward a vacuum with $N_{H}=0$. As we have seen in Section II, fermion number is not violated if the configuration unwinds in this fashion. The second possibility is that at late times the configuration dissipates toward a vacuum with $N_{H}$ unchanged from its initial value of \pm 1 . In other words, the Higgs field does not unwind but instead the gauge field "winds up". We saw in Section II that fermions are produced in the background of a solution which evolves in this way. Without $\mathcal{C P}$ violation, for every $N_{H}=+1$ configuration which relaxes in a baryon producing fashion there is an $N_{H}=-1$ configuration which produces anti-baryons. With the inclusion of the $\mathcal{C P}$ violating operator $\mathcal{O}$, the hope is that there will be some configurations which produce baryons whose $\mathcal{C P}$ conjugate configurations relax to the $N_{H}=0$ vacuum without violating baryon number.

We now pause to say a few words about the dynamical context in which we wish to use the scenario just described. We imagine that the (thin) bubble wall has just passed, leaving in its wake the configuration we are looking at, but that this configuration has 
not yet had time to relax to equilibrium. Our goal is a qualitative understanding of the dynamics of this relaxation. A first order electroweak phase transition can be characterized by the change in the gauge invariant quantity $\left\langle\sigma^{2}\right\rangle$. If we renormalize $\left\langle\sigma^{2}\right\rangle$ such that it is equal to $v^{2}$ at zero temperature, then for a strongly first order phase transition it is close to $v^{2}$ just below $T_{c}$ in the low temperature phase, and is much smaller just above $T_{c}$ in the high temperature phase. This is a slight motivation for considering initial configurations in which $\sigma=v$ throughout space, even though this is in reality not a good description of the non-equilibrium configurations left in the wake of the wall and is in fact not maintained during the subsequent evolution. There is no justification for choosing either a spherically symmetric configuration, or one with $A_{\mu}=0$, or one which is initially at rest. We shall see that this is the Achilles heel of the whole approach.

It is worth noting that Turok and Zadrozny introduced the analysis we are describing in a somewhat different context. They considered the evolution of configurations during the passage of a thick wall, rather than after the passage of a thin wall. Instead of using the operator (1.2) to introduce $\mathcal{C P}$ violation, they began with the two Higgs doublet model and used the operator

$$
\theta \operatorname{Tr} F \tilde{F}
$$

where $\theta$ is a $\mathcal{C P}$ odd phase between the two Higgs fields. (Although the operator (3.1) is $\mathcal{C P}$ even, $\mathcal{C P}$ violating dynamics in the Higgs sector picks a sign for the change in $\langle\theta\rangle$ during the phase transition, thus communicating the $\mathcal{C P}$ violation to the gauge sector.) To avoid the complications of simulating all the fields in the two Higgs doublet model, they simplified the problem as follows. First, they considered circumstances in which $\langle\theta\rangle$ was spatially uniform but changes linearly in time during the passage of the bubble wall. That is, they replaced $\theta(\mathbf{x}, t)$ in (3.1) by $\theta(t)=c t$ with $c$ a constant. Second, they chose to consider the effect of this forcing term, now treated as externally imposed, on just the standard model fields. That is, they found solutions to the equations of motion obtained from a Lagrangian given by (2.1) with the addition of the term $(c t \operatorname{Tr} F \tilde{F})$. Since this Lagrangian depends explicitly on time, 
there is no conserved energy in their problem. If we wanted to explore the effects of the $\mathcal{C P}$ violating operator (1.2) used in this paper as $\left\langle\sigma^{2}\right\rangle$ changes during the passage of the wall, we could follow precisely the same strategy. Instead, we wish to treat the non-equilibrium conditions after the passage of a thin wall. Therefore, we simply use a Lagrangian given by (2.1) plus (1.2). This means that energy is conserved (to better than half a percent in our numerical simulations) during the evolution of the gauge and Higgs fields.

We wish to solve the equations of motion obtained from the action (2.1) augmented by the addition of the operator (1.2). We work in the spherical ansatz [30 in which all gauge invariant quantities are functions only of $r$ and $t$, and solve the equations numerically. In this paper, we do not describe our numerical methods in any detail; they are standard and are similar to those of Ref. [29]. We also do not present our solutions in full. Rather, we describe them only in sufficient detail to demonstrate that it is very difficult to follow this approach to completion. Let us first consider initial conditions of the form (2.11) with $\eta(r)$ of (2.12) given by

$$
\eta(r)=-\pi\left[1-\tanh \left(\frac{r}{R}\right)\right]
$$

where $R$ is a constant parametrizing the size of the configuration. This configuration satisfies the boundary condition (2.7) and has Higgs winding number $N_{H}=+1$, and we use it as the initial condition for the equations of motion, setting all time derivatives to zero at $t=0$. For the moment, we set $b=0$ in (1.2) and do not introduce $\mathcal{C P}$ violation. In agreement with Turok and Zadrozny, we find that there is a critical value of $R$, which we call $R_{c}^{+}$, defined as follows. For all $R<R_{c}^{+}$the configuration evolves toward a vacuum configuration with $N_{H}=0$. No fermions are produced in this background. For all $R>R_{c}^{+}$, the configuration evolves toward a vacuum configuration with $N_{H}=+1$, and fermions are produced. The values of $R_{c}^{+}$which we obtain in simulations with several different values of $m_{H} / m_{W}$ are in quantitative agreement with those obtained in Ref. [6]. Repeating this exercise beginning with $\eta(r)$ given by -1 times that in (3.2), that is beginning with the $\mathcal{C P}$ conjugate configuration having $N_{H}=-1$, we find an analogously defined $R_{c}^{-}$. As we have 
not yet introduced any $\mathcal{C P}$ violation, we necessarily find $R_{c}^{-}=R_{c}^{+}$. We now repeat the entire procedure with $b \neq 0$, that is with $\mathcal{C P}$ violation present. Although at this point quantitative comparison with Ref. [6] is no longer possible because of the differences described above, we do in fact find $R_{c}^{-} \neq R_{c}^{+}$. Unfortunately, this is not the end of the story.

We now investigate slightly more general initial conditions. Consider initial configurations exactly as above except that the time derivative of $\sigma$ is nonzero and is given by

$$
\dot{\sigma}(r)=\gamma v^{2}[1-\tanh (r / R)]
$$

with $\gamma$ some constant. We now find that for some values of $\gamma, R_{c}^{-}<R_{c}^{+}$whereas for other values of $\gamma, R_{c}^{-}>R_{c}^{+}$. This dooms an analysis in terms of the single parameter $R$. Clearly, a more general framework is needed.

Consider a family of initial configurations with $N_{H}=+1$, much more general than we have considered to this point, parametrized by a set of parameters $\beta_{i}$. To this point, we have introduced two such parameters, $R$ of (3.2) and (3.3) and $\gamma$ of (3.3). More generally, we must consider other profile functions for $\eta$ and $\dot{\sigma}$ and must allow for initial configurations in which $\sigma$ varies with $r$ and $A_{\mu}$ and $\dot{A}_{\mu}$ are nonzero. More generally still, one would want to go beyond the spherical ansatz. Of course eventually one wants to work towards an analysis involving an infinite set of $\beta$ 's, but it seems reasonable to start with some finite set $\beta_{i}$. For any fixed $b$, define a function $F^{+}\left(\beta_{1}, \beta_{2}, \ldots\right)$ which has the following properties. $F^{+}\left(\beta_{1}, \beta_{2}, \ldots\right)>0$ for all points in $\beta$-space which describe configurations which evolve toward the $N_{H}=+1$ vacuum thereby producing fermions, and $F^{+}\left(\beta_{1}, \beta_{2}, \ldots\right)<$ 0 for all points in $\beta$-space describing configurations which evolve towards the $N_{H}=0$ vacuum. If we only consider initial configurations described by (2.11), (2.12) and (3.2) which are parametrized by the single parameter $R$, then we can take $F^{+}(R)=R-R_{c}^{+}$. Completely analogously, we define a function $F^{-}\left(\beta_{1}, \beta_{2}, \ldots\right)$ such that the hypersurface $F^{-}\left(\beta_{1}, \beta_{2}, \ldots\right)=0$ divides the $\beta$-space of $N_{H}=-1$ configurations into those which evolve towards the $N_{H}=-1$ and $N_{H}=0$ vacua.

In the absence of $\mathcal{C P}$ violation, $F^{+}\left(\beta_{1}, \beta_{2}, \ldots\right)=0$ and $F^{-}\left(\beta_{1}, \beta_{2}, \ldots\right)=0$ define the same hypersurface. In this case, imagine allowing a $\mathcal{C P}$ symmetric ensemble of configurations with 


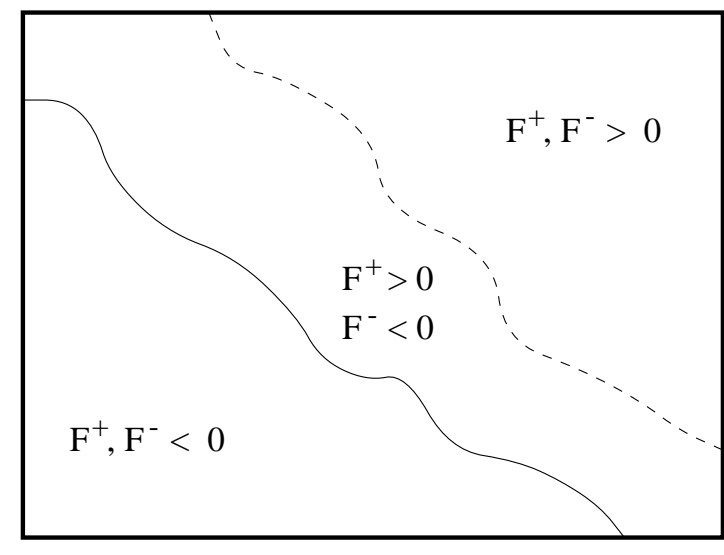

(a)

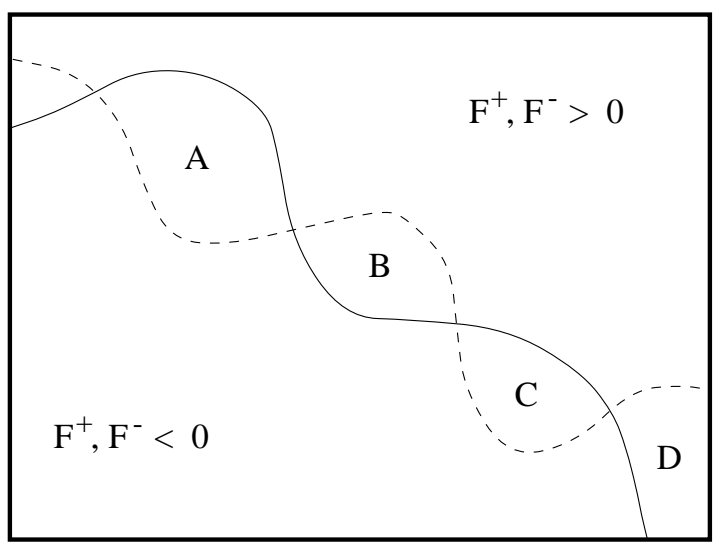

(b)

FIG. 1. Sketch of two qualitatively different possible behaviors of the critical surfaces $F^{+}=0$ (represented by a solid line) and $F^{-}=0$ (represented by a dashed line) in the space $\left(\beta_{1}, \beta_{2}, \ldots\right.$ ) describing initial configurations.

$N_{H}=+1$ and $N_{H}=-1$ to evolve. (By $\mathcal{C P}$ symmetric we mean that the probability for finding a particular $N_{H}=+1$ configuration in the ensemble is equal to that for finding its $\mathcal{C P}$ conjugate $N_{H}=-1$ configuration.) Since the configurations which anomalously produce fermions are exactly balanced by those which anomalously produce antifermions, the net fermion number produced after relaxation would be zero. We wish to investigate the behavior in the presence of the $\mathcal{C P}$ violating term $\mathcal{O}$ of (1.2). The hope is that $\mathcal{O}$ will affect the dynamics of $N_{H}=+1$ configurations and $N_{H}=-1$ configurations in qualitatively different ways and that after relaxation to vacuum a net fermion number will result, even though the initial ensemble of configurations was $\mathcal{C P}$ symmetric. With $b \neq 0$ we have seen that the two hypersurfaces $F^{+}=0$ and $F^{-}=0$ are indeed distinct. The configurations represented by points in $\beta$-space between the two hypersurfaces yield a net baryon asymmetry. There are two qualitatively different possibilities, however, which we illustrate schematically in Figure 1. In Figure 1a, the hypersurfaces $F^{+}=0$ and $F^{-}=0$ do not cross. The sign of $b$ has been chosen such that we produce net baryons in the region between the two surfaces. In Figure 1b, the hypersurfaces cross and we produce net baryons in regions $B$ and $D$, and net antibaryons in regions $A$ and $C$. If the hypersurfaces do not cross, as in Figure 1a, then a simple estimate of the fraction of configurations which yield a net baryon 
asymmetry is possible. This fraction would be proportional to the separation between the two hypersurfaces measured in any direction in $\beta$-space with a component perpendicular to the hypersurfaces - for example, it would be proportional to $\left(R_{c}^{+}-R_{c}^{-}\right)$- and it would be proportional to $b / M^{2}$, the coefficient of $\mathcal{O}$. Unfortunately, we have seen that when we consider the two parameter space of $(R, \gamma)$ the hypersurfaces $F^{+}=0$ and $F^{-}=0$ do in fact cross. Thus, in the more general space $\left(\beta_{1}, \beta_{2}, \ldots\right)$ the picture cannot look like that sketched in Figure 1a and must look like that sketched in Figure 1b.

There are other indications that life is complicated, as in Figure 1b. We have found that the evolution of configurations near the $F^{+}=0$ and $F^{-}=0$ surfaces is in some cases extremely complicated. For example, there are instances in which just to one side of $F^{+}=0$, the configuration changes its winding number from $N_{H}=1$ to $N_{H}=0$ to $N_{H}=1$ to $N_{H}=0$ to $N_{H}=1$, having a zero of the Higgs field at four different times, before finally settling toward the $N_{H}=1$ vacuum, whereas just on the other side of $F^{+}=0$ the configuration goes through three zeroes of the Higgs field before settling towards the $N_{H}=0$ vacuum. This behavior, also seen by Turok and Zadrozny, suggests that the location of the $F^{+}=0$ surface in $\beta$-space depends on details of a complicated nonlinear evolution, and so must be a highly nontrivial function of many of the parameters specifying the initial configuration. It would be very nice to find a simple characterization of the $F^{+}=0$ and $F^{-}=0$ surfaces in terms of only one or a few parameters, but this seems very unlikely. Hence, although the dynamics of the unwinding of topological configurations after the phase transition in the presence of the operator $\mathcal{O}$ may lead to a baryon asymmetry, we see no way to make a simple analytical estimate of this asymmetry. This may well be a valid way of looking at the microphysics of electroweak baryogenesis, but it seems that large scale $3+1$ dimensional numerical simulations of the kind recently pioneered by Moore and Turok [31] (but including $\mathcal{C P}$ violation via (1.2) and working in a setting in which the bubble walls are thin and rapidly moving) are required in order to estimate the contribution to the BAU. In Section V, we will return to a brief discussion of the large scale numerical simulations which seem necessary. Before that, in the next section, we explore a different attempt at obtaining a semi-analytical estimate of the magnitude of the effect. 


\section{KICKING CONFIGURATIONS ACROSS THE BARRIER}

Let us now turn to what may seem initially to be a somewhat orthogonal discussion of the physics of local electroweak baryogenesis, following that of Dine, Huet, Singleton, and Susskind [7]. In a sense, this discussion is more general than that of the previous section, because it attempts to treat baryon number violating processes of a type more general than the unwinding of winding number one configurations. On the other hand, the treatment of these more general processes is, of necessity, greatly over-simplified. The complications we encountered in the previous section are real, but they seem not to appear in the treatment of this section, and we are able to obtain an estimate for the baryon asymmetry generated. A priori this could either mean that the method extracts the essence of the matter or that it sweeps important physics under the rug. The truth is somewhere in between these two extremes. We give a discussion of the physics under the rug, and argue that the estimate we obtain should be seen as an upper bound on the baryon asymmetry generated by local electroweak baryogenesis in the thin wall scenario. Although we follow Ref. [7] to some extent, our discussion does not exactly parallel theirs and we shall note the points where we differ as we come to them.

In the high temperature phase, baryon number violating processes are not exponentially suppressed. The barrier crossing configurations typically [19,32 have sizes given by the magnetic correlation length

$$
\xi \sim\left(\alpha_{W} T\right)^{-1}
$$

We will think of dividing space up into cells of this size, and looking at configurations cell by cell. The energy in gauge field oscillations with wavelength $\xi$ is of order $T$, but the total energy in a cell is much larger, as it is presumably of order $T^{4} \xi^{3}$. Indeed, this energy is much

larger than the sphaleron energy, which is $E_{\mathrm{sph}} \sim v / g$. Most of the energy is in oscillations of the gauge and Higgs fields on length scales shorter than $\xi$. These configurations are crossing the barrier between vacua via regions of the barrier far above the lowest point on the barrier, 
that is far above the sphaleron, and they look nothing like the sphaleron. It was initially thought [19 that in each cell of volume $\xi^{3}$, the sphaleron barrier was crossed once per time $\xi$, leading to a baryon number violation rate per unit volume of the form (1.1) with $\kappa \sim 1$, in agreement with numerical simulations [33]. A recent analysis [32] in fact suggests that the time it takes for a configuration in a given cell to cross the barrier is of order $\xi / \alpha_{W}$ leading to $\kappa \sim \alpha_{W}$. We now consider what happens when the bubble wall hits the configurations just described.

Consider the configuration in one cell. It traverses a path through configuration space, which we parametrize by $\tau$. Dine et al. consider the special case in which this path is the path in configuration space which an instanton follows as a function of Euclidean time $\tau$, but this is not essential, and it is clear that they were thinking of more general circumstances also. The configurations discussed in Section III can be seen as special cases of those described here. The energy of the configuration has a maximum at some $\tau$ (at which the configuration crosses the barrier) which we define to be $\tau=0$. Following Dine et al., we now write down a Lagrangian which is intended to describe the dynamics of $\tau$ as a function of time for $\tau$ near $\tau=0$ :

$$
\mathcal{L}(\tau, \dot{\tau})=\frac{c_{1}}{2 \xi} \dot{\tau}^{2}+\frac{c_{2}}{2 \xi^{3}} \tau^{2}+\frac{c_{3}}{\xi} \frac{b}{M^{2}} \sigma^{2} \dot{\tau} .
$$

In this expression, $c_{1}, c_{2}$, and $c_{3}$ are dimensionless constants, different for each of the infinitely many possible barrier crossing trajectories. The factors of $\xi$ have been put in by dimensional analysis treating $\tau$ as a quantity of dimension -1 . (We shall see that rescaling $\tau$ by a dimensionful constant does not change the final result.) This Lagrangian should be seen as the first few terms in an expansion in powers of $\tau$ and $\dot{\tau}$. Because we have assumed that $\tau=0$ is a maximum of the energy as a function of $\tau$, no odd powers of $\tau$ can appear. In the absence of $\mathcal{C P}$ violation, there can be no odd powers of $\dot{\tau}$, since they make the dynamics for crossing the barrier from left to right different than from right to left. The $\operatorname{Tr} F \tilde{F}$ in the operator $\mathcal{O}$ includes a term which is proportional to the time derivative of the ChernSimons number, and this means that $\mathcal{O}$ must contribute a term in $\mathcal{L}$ which is linear in $\dot{\tau}$. It 
is obviously quite an over-simplification to treat barrier crossing as problem with one degree of freedom. As we saw in Section III, the complete dynamics can be very complicated, even for relatively simple initial conditions. We will return to this point, but for now we forge ahead with (4.2).

The momentum conjugate to $\tau$ is given by

$$
p=\frac{c_{1}}{\xi} \dot{\tau}+\frac{c_{3}}{\xi} \frac{b}{M^{2}} \sigma^{2}
$$

and the Hamiltonian density is therefore

$$
\mathcal{H}=\frac{\xi}{2 c_{1}}\left(p-\frac{c_{3}}{\xi} \frac{b}{M^{2}} \sigma^{2}\right)^{2}-\frac{c_{2}}{2 \xi^{3}} \tau^{2} .
$$

Before considering the thin wall case of interest in this paper, it is worth pausing to consider the thick wall limit in which $\left\langle\sigma^{2}\right\rangle$ is changing slowly and other quantities evolve adiabatically in this slowly changing background. A reasonable assumption is that the variables $(\tau, p)$ are Boltzmann distributed with respect to the Hamiltonian (4.4) at each instant, treating $\sigma^{2}$ as approximately constant. This implies that the distribution of $p$ is centered at

$$
p_{0}=\frac{c_{3}}{\xi} \frac{b}{M^{2}} \sigma^{2} .
$$

However, from (4.3) we see that this means that the velocity $\dot{\tau}$ is Boltzmann distributed with center $\dot{\tau}=0$. Thus, the presence of the $\mathcal{C P}$ violating operator (1.2) does not bias the velocity of trajectories in configuration space in the thick wall limit. This conclusion disagrees with that of Ref. [7]. There is nevertheless an effect. Integrating the third term in (4.2) by parts, one obtains a term linear in $\tau$ proportional to the time derivative of $\sigma^{2}$. This changes the shape of the potential energy surface in configuration space during the passage of the wall, and yields an asymmetry. In this limit, in which the wall is thick and departure from equilibrium is small, the problem is much more easily treated in the language of spontaneous baryogenesis [4]:7,8] - the operator $\mathcal{O}$ acts like a chemical potential for baryon number.

We now turn to the thin wall case. Immediately after the wall strikes, the fields are not yet in equilibrium. As we saw in the previous section, life is complicated. The idea of this 
section, however, is to use an impulse approximation to estimate the kick which $\dot{\tau}$ receives as the wall passes, and from this to estimate the baryon asymmetry that results. The equation of motion for $\tau$ obtained from (4.2) is

$$
\ddot{\tau}=\frac{c_{2}}{c_{1} \xi^{2}} \tau-\frac{c_{3}}{c_{1}} \frac{b}{M^{2}} \frac{d}{d t} \sigma^{2} .
$$

During the passage of a thin wall, the first term on the right hand side can be neglected relative to the second. Making this impulse approximation, we find that the passage of the wall kicks $\dot{\tau}$ by an amount

$$
\Delta \dot{\tau}=-\frac{c_{3}}{c_{1}} \frac{b}{M^{2}} \Delta \sigma^{2}
$$

where $\Delta \sigma^{2}$ is the amount by which $\sigma^{2}$ changes at the phase transition. The kick $\Delta \dot{\tau}$ has a definite sign. Thus, in the thin-wall limit, the distribution of the velocities in configuration space of barrier crossing trajectories is biased and a baryon asymmetry, whose magnitude we now discuss, results.

If $\Delta \dot{\tau}$ is large compared to $\dot{\tau}_{0}$, the velocity the configuration would have had as it crossed $\tau=0$ in the absence of the action of the wall, then $\Delta \dot{\tau}$ will kick the configuration over the barrier in the direction it favors, and will produce, say, baryons rather than anti-baryons. If $\Delta \dot{\tau}$ is small compared to $\dot{\tau}_{0}$, it will have no qualitative effect. The fraction of the distribution of configurations with $\dot{\tau}_{0}<\Delta \dot{\tau}$ is proportional to $\Delta \dot{\tau}$. (Note that had we taken $\tau$ to have dimension other than -1 in (4.2), various powers of $\xi$ would have run through the calculation until this point when they would have cancelled in computing the fraction of the distribution of $\dot{\tau}$ affected by the wall.) Note that in this calculation it was not necessary for $\tau$ to be precisely at $\tau=0$ when the wall hits. It was only necessary for $\tau$ to be close enough to $\tau=0$ that the Lagrangian (4.2) is a good approximation. It is difficult to quantify what fraction of configurations satisfy this criterion of being "close enough to $\tau=0$ ", so we simply parametrize our ignorance by calling this fraction $f$, for fudge factor. It is worth noting that $f$ does not depend on the time it takes configurations to traverse the barrier. As discussed at the beginning of the section, this is now thought to be of order $\xi / \alpha_{W}$. Making this time, 
say, longer just makes the time during which the configuration is "close enough to $\tau=0$ " longer and need not affect $f$. Tabling further discussion of $f$ momentarily, we estimate the net number density of baryons produced as

$$
n_{B} \sim \Delta \dot{\tau} f \xi^{-3}
$$

where we have absorbed the constants $c_{i}$ into $f$.

At the time of the electroweak phase transition, the entropy density of the universe is $s \sim 45 T^{3}$, and so we obtain!

$$
\frac{n_{B}}{s} \sim f \frac{\alpha_{W}^{3}}{45} \frac{b}{M^{2}} \Delta \sigma^{2}
$$

The size of the effect clearly depends on $\Delta \sigma^{2}$. It has been suggested [7] that $\Delta \sigma^{2}$ corresponds to increasing $\sigma^{2}$ up to that value at which baryon number violating processes become exponentially suppressed in thermal equilibrium. Whereas in the thick wall case, baryon number violating processes stop when $\sigma^{2}$ reaches this value, this is not the case in the thin wall scenario. In this setting, thermal equilibrium is not maintained even approximately, and we see from the above discussion that what matters is the net change in $\sigma^{2}$ as the wall passes. (If we assume that $\sigma^{2}$ changes arbitrarily rapidly as we did by using the impulse approximation above, then the final answer can only depend on the total jump in $\sigma^{2}$ and cannot depend on the value of $\sigma^{2}$ at which equilibrium baryon number violation ceases.) Once one picks an extension of the standard model which makes the transition strongly first order, one can compute $\Delta \sigma^{2}$. Here, we will simply take $\Delta \sigma^{2}=v^{2} / 2$, which is approximately what is obtained in the minimal standard model with a $35 \mathrm{GeV}$ Higgs mass [21]. Putting it all together, we find

$$
\frac{n_{B}}{s} \sim f\left(1 \times 10^{-9}\right) b \frac{(5 \mathrm{TeV})^{2}}{M^{2}} .
$$

\footnotetext{
${ }^{1}$ This result agrees with that of Ref. [7], although our discussion and theirs are somewhat different. In comparing to Ref. [7] note that they take $b \sim \alpha_{W}$.
} 
If we take, for example, $b \sim \alpha_{W}$ and $M \sim 1 \mathrm{TeV}$, the bound (1.5) can be satisfied and (4.10) suggests that a cosmologically relevant BAU may be generated. We see that if $\mathcal{C P}$ violation is introduced via the operator $\mathcal{O}$ with a coefficient $b / M^{2}$ satisfying (1.5), and if the bubble walls are thin, then the contribution to the baryon asymmetry of the universe from local electroweak baryogenesis can be at an interesting level so long as the fudge factor $f$ is not smaller than about a tenth.

We have reached the estimate (4.10) by arguing that $n_{B}$ must be proportional to $\left(b / M^{2}\right) \Delta \sigma^{2}$, arguing by dimensional analysis that it must be proportional to $\xi^{-3}$, arguing that the time it takes configurations to cross the barrier in equilibrium in the high temperature phase is not relevant, and lumping our remaining ignorance into $f$. We now turn to a discussion of what goes into $f$, and how the treatments of Sections III and IV are related. There are many contributions to $f$, since the treatment leading to the estimate (4.10) is greatly over-simplified. First, there are the constants $c_{i}$, which of course differ for the different configurations in different cells of volume $\xi^{3}$, and must somehow be averaged over. Second, using the impulse approximation is not really justified. In reality, the wall does not have zero thickness. More important, even if the wall is thin, the time during which it can affect a configuration of size $\xi$ is at least $\xi$. Third, as we have already mentioned, the treatment in terms of the Lagrangian (4.2) only has a chance of capturing the physics near $\tau=0$, and it is not at all clear what fraction of configurations satisfy this. Configurations which happen to be farther away from the crest of the ridge between vacua when the wall hits do receive a kick from the wall. However, even if this kick is large, it may not be in a suitable direction in configuration space to be effective. Configurations far from $\tau=0$ can contribute to $n_{B}$, but their contribution is hard to compute, because there is no way to reduce the problem to one of one degree of freedom far from $\tau=0$. Fourth, even near the crest of the ridge for a given trajectory the problem does not really reduce to one degree of freedom. For the configurations of interest, $\sigma$ is a function of space and time and the operator $\mathcal{O}$ and the bubble wall conspire to affect its dynamics. We have attempted to describe the effect by treating $\sigma$ as constant in space and time on either side of the wall and 
only changing at the wall. This is a caricature at best. Fifth, by now the problem should be sounding more like that of Section III, and we must face up to the specific difficulties discussed there. After the wall has passed, the fields are not yet in thermal equilibrium and their dynamics is complicated. This may in fact yield a further contribution to $n_{B}$. It may also, however, negate some of the contribution estimated in (4.10) because some configurations kicked across the barrier in one direction by the passage of the wall may at a later time wander back across the barrier whence they came. As we discovered in Section III, an estimate of the magnitude of these sorts of effects is difficult even for the restricted class of configurations we considered there. To sum up, $f$ is almost certainly less than 1 . Hence, it would be best to use (4.10) as an upper bound on $n_{B} / s$, rather than as an estimate.

\section{CONCLUDING REMARKS}

If the electroweak phase transition is strongly first order, it is possible that the observed baryon asymmetry of the universe may have been generated by non-equilibrium processes occurring as bubble walls sweep through the plasma. In general, there will be contributions to the asymmetry both from local baryogenesis and from nonlocal baryogenesis. In the last few years, much effort has been devoted to computing the contribution from nonlocal effects [5.9 14]. Recently, the work of Moore and Turok 31] has made it clear that the computational resources now available make a large scale numerical treatment of local baryogenesis a possibility. Our goal in this paper was to reconsider two possible routes to a semi-analytic estimate of the magnitude of the effect. If the bubble walls are thick, conditions remain close to thermal equilibrium during the passage of the wall, and the nonequilibrium physics can be captured by assigning nonzero chemical potentials to various quantum numbers including baryon number. Analytic estimates for the BAU produced in this setting exist in the literature [7,8] and the need for a numerical treatment is not pressing. If the bubble walls are thin, however, or if (as is no doubt the case) they are comparable in thickness to other length scales in the problem, the situation is unsettled. We have reanalyzed the problem of local baryogenesis in the thin wall limit using two different semi-analytical approaches [6,7]. 
The method of Dine et al. does yield an estimate (4.10), but the uncertainties parametrized by $f$, particularly those highlighted by the difficulties which prevented us from obtaining an estimate via the method of Turok and Zadrozny, mean that this should be viewed at best as an upper bound. A large scale numerical treatment seems necessary.

Moore and Turok [31] have recently taken a big step in this direction. They have performed $3+1$ dimensional classical simulations in which a bubble wall moves through a box converting the high temperature phase to the low temperature phase. To date, they have focused more on computing quantities like the wall thickness, the wall velocity, the surface tension, and the drag on the wall and have only begun their treatment of local electroweak baryogenesis. To this point, they have introduced $\mathcal{C P}$ violation only by "mocking up" the effects of (1.2) by first computing the average wall profile $\langle\sigma\rangle(z)$ for an ensemble of walls, and then doing a simulation in which one measures the distance of a given point to the nearest bubble wall and adds a chemical potential for Chern-Simons number at that point proportional to the spatial derivative of the average wall profile at that distance. This chemical potential is only nonzero on the wall, as it would be if it were proportional to $\frac{d}{d t} \sigma^{2}$ for a moving wall. Nevertheless, by imposing the chemical potential as an external driving force instead of simply introducing (1.2) in the Lagrangian and letting the dynamics do their thing self-consistently, one risks missing a lot of the difficulties (and potential effects) we have discussed in Section III and at the end of Section IV. The simulations of Moore and Turok suggest that a large scale numerical assault on the problem of local electroweak baryogenesis is now possible; the difficulties we have discussed which prevent us from obtaining a reliable semi-analytic estimate of the magnitude of the effect show that it is necessary.

Although we have taken considerable care to describe the failings of (4.10), even if we use it only as an upper bound it is interesting in the following sense. Let us assume that whatever $\mathcal{C P}$ violation is introduced in order to make baryogenesis possible can be parametrized by the $\mathcal{C P}$ violating operator $\mathcal{O}$ of $(1.2)$. Combined with the experimental bound (1.5) on the coefficient of $\mathcal{O}$, the result (4.10) shows that if the experimental sensitivity to the electric dipole moment of the electron or the neutron can be improved by about an order 
of magnitude, and if these experiments continue to yield results consistent with zero, then the baryon asymmetry of the universe produced by local electroweak baryogenesis is smaller than that observed, even if future numerical simulations were to demonstrate that $f$ is as large as 1 .

\section{ACKNOWLEDGMENTS}

We would first like to thank Eddie Farhi for fruitful collaboration during the early stages of this project, and in particular for his invaluable contributions to Section II. We would also like to thank the following people for helpful comments and discussions: Peter Arnold, Robert Brandenberger, Sean Carroll, Sekhar Chivukula, Jeffrey Goldstone, Ken Johnson, Guy Moore, Lisa Randall, Bob Singleton, Neil Turok, and an anonymous referee.

The work of A.L. and M.T. was supported in part by funds provided by the U.S. Depart-

ment of Energy (D.O.E.) under cooperative research agreement \# DF-FC02-94ER40818. The work of K.R. was supported in part by the Sherman Fairchild Foundation and by the Department of Energy under Grant No. DE-FG03-92-ER40701. 


\section{REFERENCES}

[1] A. D. Sakharov, JETP Lett. 5, 24 (1967).

[2] S. Dimopoulos and L. Susskind, Phys. Rev. D18, 4500 (1978).

[3] V. A. Kuzmin, V. A. Rubakov and M. E. Shaposhnikov, Phys. Lett. B155, 36 (1985).

[4] A. G. Cohen and D. B. Kaplan, Phys. Lett. B199, 251 (1987); Nucl. Phys. B308, 913 (1988).

[5] A. G. Cohen, D. B. Kaplan and A. E. Nelson, Phys. Lett. B245, 561 (1990); Nucl. Phys. B349, 727 (1991).

[6] N. Turok and T. Zadrozny, Phys. Rev. Lett. 65, 2331 (1990); Nucl. Phys. B358, 471 (1991);

L. McLerran, M. Shaposhnikov, N. Turok and M. Voloshin, Phys. Lett. B256, 451 (1991);

[7] M. Dine, P. Huet, R. Singleton Jr. and L. Susskind, Phys. Lett. B257, 351 (1991);

M. Dine, P. Huet and R. Singleton Jr., Nucl. Phys. B375, 625 (1992).

[8] A. G. Cohen, D. B. Kaplan and A. E. Nelson, Phys. Lett. B263, 86 (1991);

A. G. Cohen and A. E. Nelson, Phys. Lett. B297, 111 (1992);

M. Dine and S. Thomas, Phys. Lett. B328, 73 (1994).

[9] A. E. Nelson, D. B. Kaplan and A. G. Cohen, Nucl. Phys. B373, 453 (1992);

A. G. Cohen, D. B. Kaplan and A. E. Nelson, Phys. Lett. B294, 57 (1992); B336, 41 (1994).

[10] G. R. Farrar and M. E. Shaposhnikov, Phys. Rev. Lett. 70, 2833 (1993); Phys. Rev D50, 774 (1994); hep-ph/9406387;

M. B. Gavela, P. Hernández, J. Orloff and O. Pène, Mod. Phys. Lett. A9, 795 (1994);

M. B. Gavela, M. Lozano, J. Orloff and O. Pène Nucl. Phys. B430, 345 (1994);

M. B. Gavela, P. Hernández, J. Orloff, O. Pène and C. Quimbay Nucl. Phys. B430, 382 (1994);

P. Huet and E. Sather Phys. Rev. D51, 379 (1995).

[11] G. F. Giudice and M. E. Shaposhnikov, Phys. Lett. B326, 118 (1994). 
[12] M. Joyce, T. Prokopec and N. Turok, Phys. Lett., B338, 269 (1994); B339, 312 (1994); Phys. Rev. D53, 2930 (1996); ibid. 2958.

[13] J. M. Cline Phys. Lett. B338, 263 (1994);

J. M. Cline, K. Kainulainen and A. P. Vischer, Phys. Rev. D54, 2451 (1996).

[14] P. Huet and A. E. Nelson, Phys. Lett. B355, 229 (1995); Phys. Rev. D53, 4578 (1996).

[15] R. H. Brandenberger, A.-C. Davis and M. Hindmarsh, Phys. Lett. B263, 239 (1991);

R. H. Brandenberger and A.-C. Davis, Phys. Lett. B308, 79 (1993);

R. H. Brandenberger, A.-C. Davis, and M. Trodden, Phys. Lett. B335, 123 (1994);

R. H. Brandenberger, A.-C. Davis, T. Prokopec and M. Trodden, Phys. Rev. D53, 4257 (1996);

T. Prokopec, R. H. Brandenberger, A.-C. Davis and M. Trodden, Phys. Lett. B384, 175 (1996);

M. Trodden, A.-C. Davis and R. H. Brandenberger, Phys. Lett. B349, 131 (1995).

[16] For a recent review, see V. A. Rubakov and M. E. Shaposhnikov, hep-ph/9603208.

[17] G. t'Hooft, Phys. Rev. Lett. 37, 8 (1976).

[18] N. S. Manton, Phys. Rev. D28, 2019 (1983);

F. R. Klinkhamer and N. S. Manton, Phys. Rev. D30, 2212 (1984).

[19] P. Arnold and L. McLerran, Phys. Rev. D36, 581 (1987).

[20] M. E. Shaposhnikov, JETP Lett. 44, 465 (1986); Nucl. Phys. B287, 757 (1987);

A. I. Bochkarev and M. E. Shaposhnikov Mod. Phys. Lett. A2, 417 (1987).

[21] K. Kajantie, M. Laine, K. Rummukainen and M. Shaposhnikov, Nucl. Phys. B466, 189 (1996).

[22] X. Zhang and B.-L. Young, Phys. Rev. D49, 563 (1994).

[23] E. D. Commins, S. B. Ross, D. DeMille and B. C. Regan, Phys. Rev. A50, 2960 (1994).

[24] K. F. Smith et al., Phys. Lett. B234, 191 (1990);

I. S. Altarev et al., Phys. Lett. B276, 242 (1992).

[25] J. Goldstone and F. Wilczek, Phys. Rev. Lett., 47, 986 (1981).

[26] E. D’Hoker and E. Farhi, Nucl. Phys. B241, 109 (1984). 
[27] R. MacKenzie and F. Wilczek, Phys. Rev. D30, 2194 (1984); ibid, D30, 2260 (1984); R. MacKenzie, F. Wilczek and A. Zee, Phys. Rev. Lett., 53, 2203 (1984).

[28] E. Farhi, J. Goldstone, S. Gutmann, K. Rajagopal and R. Singleton Jr., Phys. Rev. D51 (1995) 4561.

[29] E. Farhi, J. Goldstone, A. Lue and K. Rajagopal, Phys. Rev. D54, 5336 (1996).

[30] E. Witten, Phys. Rev. Lett. 38, 121 (1977);

B. Ratra and L.G. Yaffe, Phys. Lett. B205, 57 (1988).

[31] G. D. Moore and N. Turok, hep-ph/9608350.

[32] P. Arnold, D. T. Son and L. G. Yaffe, hep-ph/9609481;

P. Huet and D. T. Son, hep-ph/9610259.

[33] J. Ambjørn and A. Krasnitz, Phys. Lett. B362, 97 (1995);

G. D. Moore, hep-ph/9603384;

W.-H. Tang and J. Smit, hep-lat/9605016. 